\title{
Adaptive Web-Based System for Examination with Cheating Prevention Mechanism
}

\author{
Ondrej Kainz, Dávid Cymbalák, and František Jakab
}

\begin{abstract}
In this paper, the adaptive web-based system for examination with cheating prevention mechanism is presented. The idea of system was created based on the need to provide impartial evaluation during testing of learners - statistics of cheating during exams from the last decade is rather alarming however not surprising. With the dawn of new technologies cheating is becoming much easier than it used to be, as a natural result certain anti-cheating mechanism has to be deployed. System itself is in nature web-based and thus enables easy and widespread access. Approaches that enable automatic monitoring of learner during an examination were following and enhancing those in previously created systems. As a communication technology over the internet or intranet was utilized WebRTC which enabled to incorporate together various live monitoring capabilities - video, audio, screen of computer. Another feature that is in the center of interest is adaptive selection of questions. This selection is automatically carried out by system and it is based on answers as provided by learner. Deployment in the real environment is expected to be used as a complement that is to enhance education quality and provide automatic impartial evaluation of learner.
\end{abstract}

Index Terms-Adaptive testing, anti-cheating mechanism, distance learning, examination system, cheating prevention, MOOC, traditional learning.

\section{INTRODUCTION}

Education is one of the key elements that has significant impact on the life of an individual. Based on its qualitative characteristics it influences the quality of life from many aspects: it may determine social status or shape one's individuality. Providing high quality education to learners is one the goals that should be the virtue of every institution and its instructors. Learner with intention to cheat might be still able pass the specific exam despite the presumption that the provided education is based on solid grounds. Enforcement of anti-teaching techniques are means that are to prevent specific individual of passing through the exam.

One of the key factors when providing proper education is impartial evaluation, to achieve this goal certain premises have to be met. Following the statistics [1] over half of high school students admitted cheating, sample size of 24000 students. Another study [2] concluded that one in five university students engaged in some form of cheating,

Manuscript received April 24, 2014; revised July 10, 2014. We support research activities in Slovakia / This project is being co-financed by the European Union. Paper is the result of the Project implementation: University Science Park TECHNICOM for Innovation Applications Supported by Knowledge Technology, ITMS: 26220220182, supported by the Research \& Development Operational Program funded by the ERDF.

The authors are with the Technical University of Kosice, Slovakia (e-mail: ondrej.kainz@tuke.sk, david.cymbalak@tuke.sk,frantisek.jakab@tuke.sk). sample size of 80000 students. These results are not surprising but rather expected. In this paper we address and implement several of anti-cheating features and expect promising results when implemented in the real environment. Our solution is built on web technology that provides wide availability, easy access and user friendly interface while providing anti-cheating capabilities. Developed tool was designed to enable checking of learner's activity in various environments - it is suitable for traditional education and as well as for distance education, e.g. Massive Open Online Course (MOOC). Principal idea in this stage of project is to provide a tool for cheating detection during exams, adaptive selection of questions both of these are to result in the impartial evaluation.

\section{RELATED WORK}

Systems with anti-cheating capabilities are not very frequent, as a basic feature of systems with such capabilities is commonly randomization of questions stored in database. Monitoring of learner's activity and evaluation whether cheating is detected is fully in the hands of instructor. As the data below clearly shows, frauds are pretty common and instructor may be unable to prevent them.

Research in the field of academic cheating and frauds was in the focus of [3], authors conducted an analysis of attitudes towards frauds on sample of 1527 engineering students where merely 5.4 percent of students reported that they have never observed fraud.

New approach for online examination was proposed by [4]. Authors aimed to prevent learners from direct cheating through the usage of prohibited tools (book, computer or phone) and direct guidance. Solution consisted of identification of user through the fingerprint reader that also served as a tool for motion tracking - as a device was used fingerprint scanner enabled light pan, opting pad and mice. As a next prevention mechanism was utilized audio streaming of questions that were given to learners, this approach allowed strict timing. Isolated and adjusted system environment was enabled by Live OS medium that was loaded after computer booting process. Authors in [5] developed online examination system with two strategies that focus on anti-cheating: automatic generation of examination paper and second disabling features of Windows operation system - e.g. taskbar, hotkeys, right-mouse click etc. In this way learner will be prevented to misuse computer.

Both of just presented approaches have certain drawbacks. In developed tool we propose such solution that is to mitigate all of them, e.g. reading answers from neighboring screen, using prohibited aids or communicating with neighboring student. 


\section{SOLUTION PROPOSAL}

\section{A. Motivation}

"If students find out that others are more successful in their works through cheating, a kind of demoralization will be developed, without really talking about it. And if cheating is not got under control, it provokes a feeling of injustice among individuals. In short, academic dishonesty should not be considered as a victimless crime [6]."

Principal drive for development of tool with anti-cheating capabilities is resulting from the above stated quote. Following the cheating methods as stated by [7] we aim to prevent learners from information exchange by using - paper, phone, e-mail, headset, Morse-code or sign language, PDA, UV ink, switching paper with another student, reading from screen of neighboring student or of course using computer device for unauthorized purposes.

\section{B. Solution Design}

Instructor as a sole entity might not be able to provide enough detection capabilities comparing to automated system. Main advantage of such system is automation, i.e. no explicit monitoring from the side of instructor is to be required. System only notifies instructor about all the exceptions and abnormalities that might occur during examination and thus provides impartial evaluation.

Examination system is aimed to be utilized in traditional and distance education. Both of these environments require different approaches to be taken during the examination itself. To enable utilization in both of these we create our system as a web-based tool. Other aspects to be considered are: availability, easy access, open-source technologies and multi-user environment. Two main capabilities are implemented: cheating prevention and adaptive testing.

Two roles are to be represented in a system: instructor and learner. In Fig. 1 is represented diagram depicting system for prevention of cheating. Instructor is able to share screen of device in order to present questions visually however questions may be represented also directly through the web interface, another option is to present questions through the voice - via the microphone or webcam with microphone. Last option of visual representation is sharing webcam image. Utilization of these techniques is arbitrary, sharing screen, directly writing questions to a web interface or real-time input via audio does not require any form of questions database and may be used for various types of examinations. Communication of instructor to learners is in a form one-to-many (corresponds to broadcast), one-to-selected (corresponds to multicast) or one-to-one (corresponds to unicast). Input for instructor is notification of abnormal activity where specific device is highlighted.

As for the learner's application running on device it requires three inputs: screen, audio input and output via headset and web-cam. All three devices are necessary for anti-cheating mechanism to be effective since threshold is set on each of these. Should the threshold be violated (e.g. detected voice, screen switching or visual change) it is to be evaluated as abnormal behavior that will result in notification of instructor - specific time delay may be set to automatically freeze the screen of device, otherwise this is done manually.
Communication between instructor and learner is enabled by chat that can be enabled only by instructor; form of communication is many-to-one (students-instructor).

Another mechanism is adaptive testing; in Fig. 2 is represented mechanism for adaptive selection of test questions and control of specific sessions. Again as for the role for instructor - has the ability to control specific session (start/stop/resume), create or delete, another option is to learner's management. Information about learners is stored in the database. Every season created in system has to have defined password.

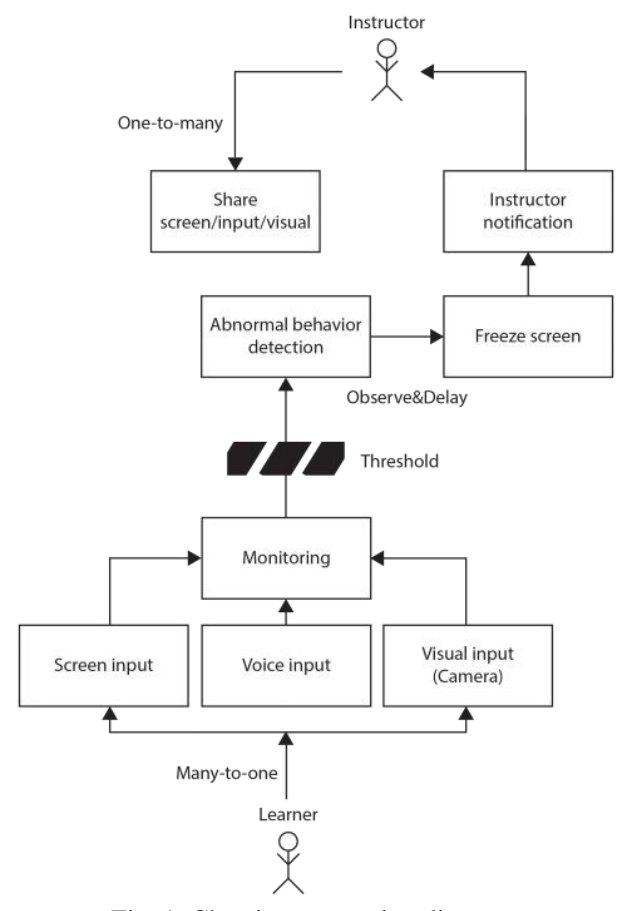

Fig. 1. Cheating prevention diagram.

After the $\log$ in into the system the learner is provided with list of available sessions (tests). If test is selected at the first are presented general questions from selected topic, i.e. question with an average level of difficulty. Based on the output learner is directed to the specific subsection - this is dependent on the results from the preceding part, only the areas where the false answers were selected is relevant for the next step. Subsequent questions are hence directly related to previously falsely answered question. However it does not mean that exclusively the questions from the specific section are presented - it is rather a ratio, where there major part is represented by subcategory and questions from other categories. Depth of the testing is dependent on the number of categories and number of difficulty levels. List of answers is grouped by similarity based on learner's output and stored in separate database for further evaluation. It should be noted that database of questions is created and predefined by instructor, i.e. number of categories, depth of difficulty etc. Instructor is responsible for the final evaluation, however test is evaluated automatically.

This system eliminates drawbacks of previously created systems - question randomization is applied only to a first set of general questions. Rest of the questions is dependent on the input as provided by learner. Usage of prohibited tools is made impossible due to monitoring of learner's surroundings via audio and video and active screen monitoring. Set of 
answers from one leaner is analyzed and compared with the rest in order to find correlation of that might indicate cheating.

Analysis of web-based technologies capable of providing such features as above presented was conducted and its results are presented in the next section.

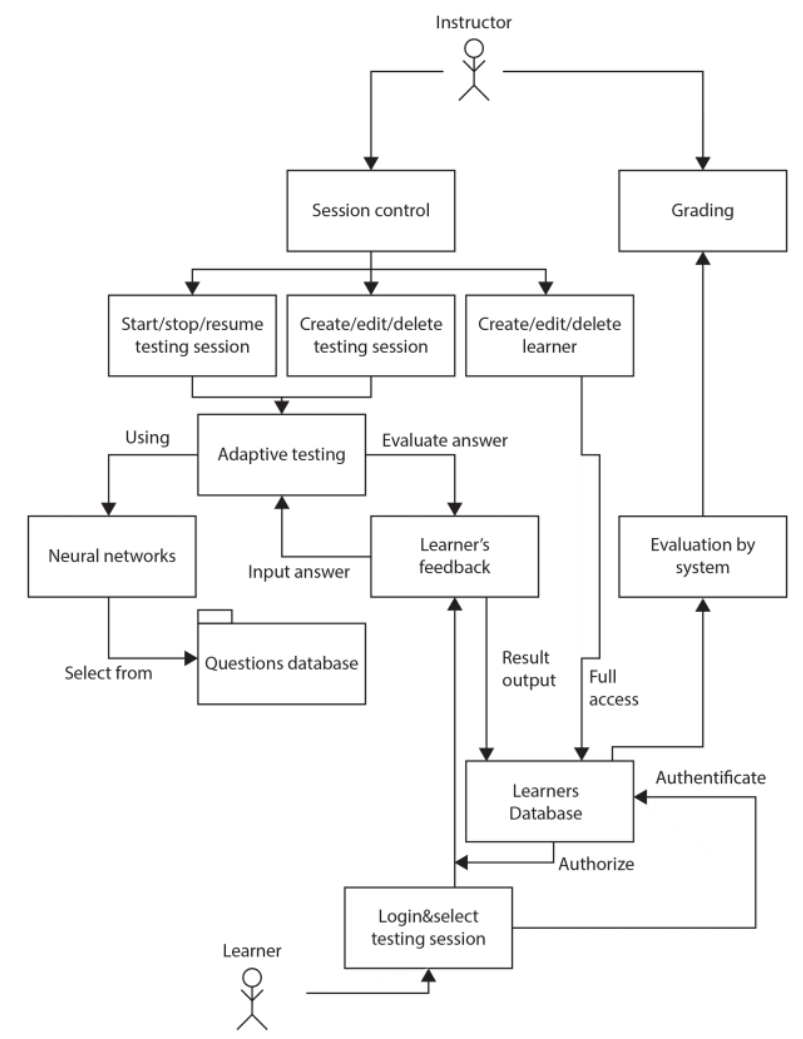

Fig. 2. Adaptive testing diagram.

\section{Communication Using WebRTC Standard}

Real-time peer-to-peer communication on the web is made possible with Web Real-Time Communication (WebRTC). This technology utilizes HTML5 and existing real-time streaming codecs and protocols for that reason eliminates the need to define new ones [8], it is rather a collection of standards, protocols and JavaScript APIs. Combination of such technologies enables peer-to-peer audio, video or data sharing between client browsers. Since it is not dependent on any third-party plugins or software it is able to take an advantage of a simple JavaScript API. In order to be fully functional the architecture of the WebRTC is made up of variety of different standards, containing both browser APIs and the application, various data formats and protocols. Responsible for definition of browser APIs is Web Real-Time Communications (WEBRTC) W3C Working Group. On the other hand Real-Time Communication in Web-browsers (RTCWEB) as a part of IETF Working Group is responsible for definition of the protocols, data formats, security, and all other necessary aspects to manage peer-to-peer communication in the browser [9].

Transport of data in WebRTC involves sending and receiving data over the network interfaces between chosen network-layer addresses on each end of the communication channel and subsequently to interact with the intermediate entities that are to handle the data however permit its modification (e.g. TURN or STUN) [10]. STUN is used as a solution providing that a media travel directly between peers, while media is redirected through the server TURN is utilized. More preferred is TURN since it is capable to traverse symmetric NATs as well. Nonetheless, STUN is beneficial when accelerating the connection between instantaneous candidates providing that users are located behind the same NAT [11].

Audio and video processing capabilities, new application APIs or support for various kind of new network protocols, all these new functionalities in the web-based environment are required when delivering high-quality and reliable RTC applications, e.g. audio and video conferencing or peer-to-peer data exchange. In the context of WebRTC the browser itself abstracts majority of this complexity behind these main APIs: Media Stream - receiving of audio and video streams, RTC Peer Connection - communication for audio and video data, RTC Data Channel - communication for arbitrary application data [9].

Exchange of arbitrary application data in both directions between peers (Web Socket) is enabled by Data Channel. Once the RTC Peer Connection is established connected peers are enabled to exchange text or any other binary data over more than one channel. Particular Data Channel may be configured to provide reliable or partially reliable delivery for (in-order or out-of-order) messages that are being sent. Yet it allows imitation of UDP semantics if defined as unreliable, out-of-order delivery. Order of such messages and confirmation of delivery are not relevant. Another possibility is configure channel - to be partially reliable and that may be done be specifying the maximum number of retransmissions or setting a time limit, where WebRTC stack will handle the acknowledgements and time-outs. Limitations and performance characteristics are own for each channel configuration. Media streams are dynamically adjusted to adapt the conditions of the network link between the peers by WebRTC audio and video engines. Configuration and update of media constraints, e.g. frame rate, video resolution etc. may be customized by the application [9].

\section{Components Interconnection}

Two interfaces were encompassed in the experimental solution - instructor's page and learner's page (Fig. 3). Page of instructor is consisted from permanent block for viewing and rating the grouped results, definition of single/multi-choice answers and viewing learners' results in a form of a graph, displaying the list of uploaded files with the genuineness check result, controlling for the initialization of communication with learner's side or changing the appearance of blocks for testing on side of learner. Learner's page is consisted from permanent block that enables to receive audio, video or screen and dynamic blocks for answer input, choosing to select single/multi-choice answer or upload file containing answers. Dynamic blocks are shown only if allowed by the instructor - from instructor's page. Interaction with dynamic nature for hiding and enabling the block is enabled by HTML5, AJAX, CSS3 and PHP technologies.

Chat implementation of WebRTC has been modified in order to enable manual input and dynamic display of the answers on the screen of instructor. Manually answered 
questions are simultaneously stored in groups in the database - and evaluated to detect cheating. File upload implementation by WebRTC also interacts with database, utilizing originality check.

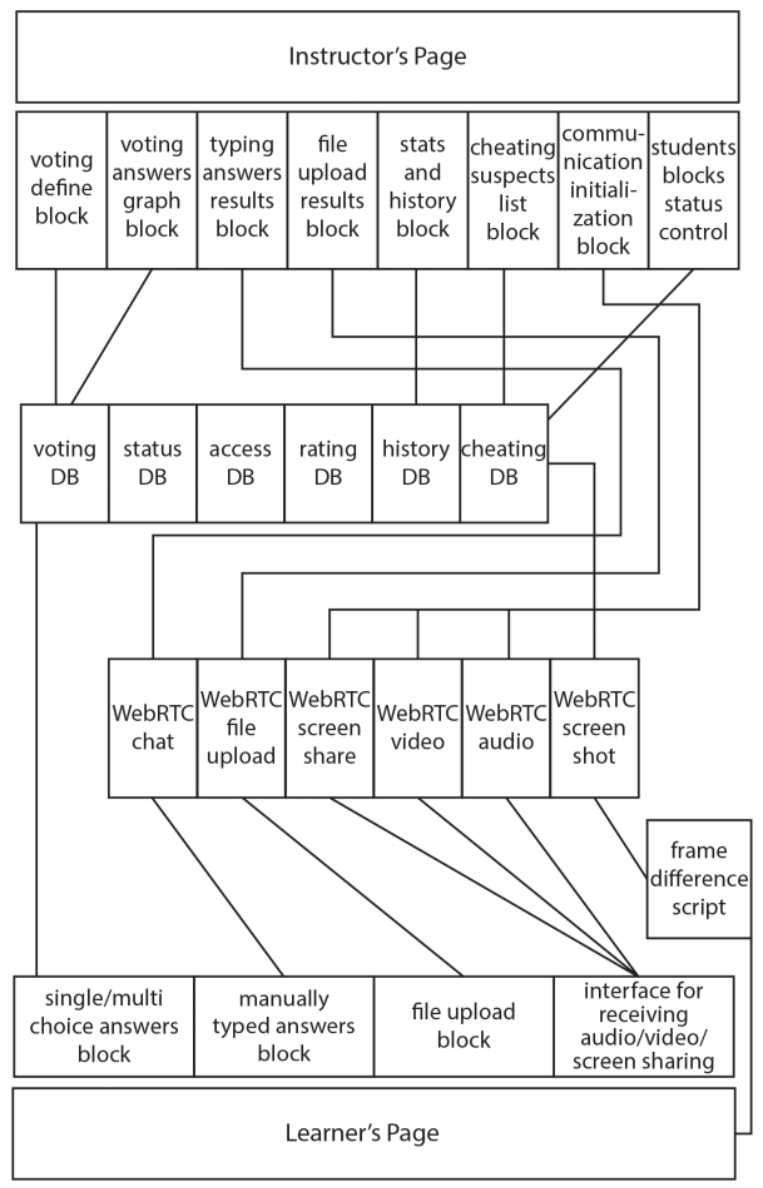

Fig. 3. Components interconnection.

Identification of learner is through ID and password, temporal ID for a specific learner to access database is optional. Screen monitoring algorithm is running on device of learner-mechanism is based on comparing frames (screen) over time. If the threshold is violated, screen is frozen and the notification is send to instructor (Fig. 4).
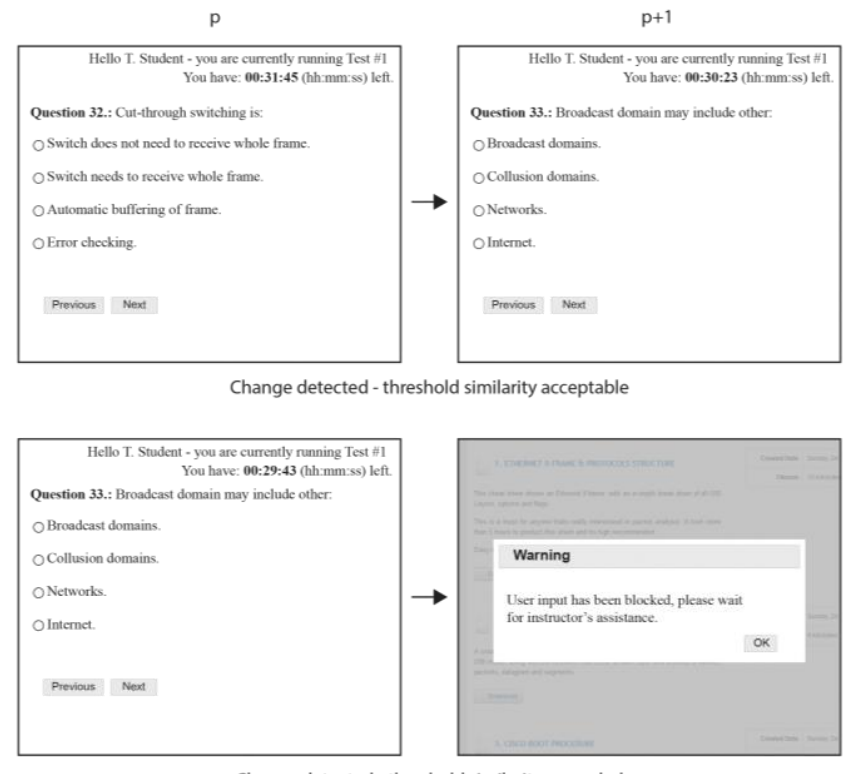

Change detected - threshold similarity exceeded

Fig. 4. Subsequent frame - screen monitoring.
Such threshold may be violated by switching between several windows, however system was set to block user only after few seconds - as the second alert is shown. Voice recording is monitored and stored locally - if detected trigger will set off threshold. Video input via webcam is to be optional, if implemented, it is to locally record learner's activity and monitor learner's position of eyes. Threshold details are to be specific for each test and optionally adjusted. The examination system is designed to ultimately prohibit learner from using computer, talking to other individual or using any other unauthorized resources that might result in cheating.

\section{CONCLUSION}

Essential part of the education is to determine the level of learner's understanding. This is however an uneasy task due to many reasons, e.g. non-impartial evaluation by instructor or learner's effort to cheat. Contemporary technology enables us to eliminate such influences by enforcing certain mechanisms into the educational practice. Following this idea we designed and implemented system for examination with anti-cheating capabilities as a rather unique solution.

System itself prevents learner from exchanging the information through the various ways - usage of prohibited aids (computer, phone, cheat sheets), talking with another learner, etc. This was achieved by automatic and constant monitoring of learner's surroundings and used device. Any situation that is evaluated as abnormal is automatically reported by system to instructor. Then it is dependent on how is the system set up - it either can automatically freeze learner's screen after specified period of time or notify instructor and put the decisions process into the hands of instructor. Another feature is adaptive selection of questions, based on output of the initial set of answers, as set by the instructor; the learner is directed to more specific questions that have direct relation to those previously answered. Questions may be provided through a visual or audio means. Enhanced privacy is expected provided that the questions are stored in audio file format, i.e. learner only hears the question or can replay it. In this way, level of difficulty to acquire the database of questions is more advanced. Utilization of system is possible in multi-environment, i.e. primary goal is to be used as a tool for traditional and distance education.

Proposed solution is expected to enhance education of the graduate learners at various kinds of institutions, though it has been primarily created to be used at the university, it may be used at primary schools or even during job interview. Due to web-based features and technologies that enabled creation of this system, it is fairly easy to implement and even more easy to use. In the near future we plan to enhance features of system to provide biometric identification of learner.

\section{REFERENCES}

[1] R. Jarc. (February 2010). Character counts: Programs: Ethics of American youth survey. Josephson Institute. [Online]. Available: http://charactercounts.org/programs/reportcard/2010/installment02_re port-card_honesty-integrity.html.

[2] D. L. McCabe, "Cheating among college and university students: A North American perspective," International Journal for Educational Integrity, vol. 1, no. 1, 2005. 
[3] P. Gama, A. Seixas, F. Almeida, P. Peixoto, and D. Esteves, "Ethics and academic fraud among higher education engineering students in Portugal," in Proc. International Portuguese Society Conf. Engineering Education, Porto, 2013, pp. 1-7.

[4] A. Trivedi, "A relevant online examination system," in Proc. International Technology for Education Conf., Mumbai, 2010, pp. 32-35.

[5] L. Siyao and G. Qianrang, "The research on anti-cheating strategy of online examination system," in Proc. International Artificial Intelligence, Management Science and Electronic Commerce Conf., Deng Feng, 2011, pp. 1738-1741.

[6] E. Khodaie, A. Moghadamzadeh, and K. Salehi, "Factors affecting the probability of academic cheating school students in Tehran," in Proc. Education and Educational Psychology Conf., 2011, vol. 29, pp. 1587-1595.

[7] B. Keresztury and L. Cser, "New cheating methods in the electronic teaching era," in Proc. Learning, Teaching and Educational Leadership Conf., 2013, vol. 93, pp. 1516-1520.

[8] P. Rodríguez, J. Cerviño, I. Trajkovska, and J. Salvachúa, "Advanced videoconferencing services based on WebRTC," in Proc. IADIS Multi Conf. Computer Science and Information Systems, 2012, pp. 180-184.

[9] I. Grigorik, High Performance Browser Networking, Sebastopol, CA: O'Reilly Media Inc., 2013.

[10] H. Alvestrand, Overview: Real Time Protocols for Brower-based Applications, Internet Engineering Task Force, 2012.

[11] K. Muaz. (2014). WebRTC Experiments and Demos. [Online]. Available: https://www.webrtc-experiment.com/.

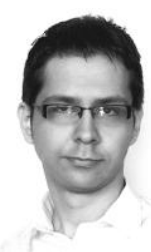

Ondrej Kainz was born in 1988. In 2013 he graduated (MSc. in applied informatics) from the Technical University in Kosice, Slovakia. Since the very same year he is a PhD student at the Department of Computers and Informatics of the Faculty of Electrical Engineering and Informatics at the Technical university of Kosice. His scientific research interests include e-learning, human-computer interfaces, computer graphics, computer networks, biological engineering and body area network.

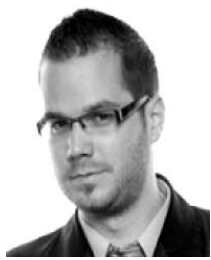

Dávid Cymbalák was born in 1987. In 2011 he graduated (MSc) at the Department of Computers and Informatics of the Faculty of Electrical Engineering and Informatics at Technical University in Kosice. Since 2011 he is a PhD student at the Department of Computers and Informatics. His scientific research is focusing on streaming technologies, e-learning solutions or object tracking methods. In addition, he also investigates questions related with the optimization of mobile access to multimedia sources.

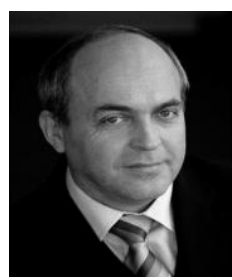

František Jakab was born in 1959. He graduated from St. Petersburg Electro Technical University (MSc. in system engineering, russia, 1984), PhD degree from Technical University of Kosice (Slovakia, 2005) \& associated professor since 2008. $\mathrm{He}$ has extensive experience in networking and utilization of ICT in education (more then 30 years teaching experience) where he established well known research centre - Computer Networks Laboratory (www.cnl.sk, in 1995). He has been an coordinator of several large international projects financed by EC, coordinator of national wide ICT projects and research grants. He act as an expert in areas: projecting of computer networks, new form of multimedia based communication (videoconferences, IP streaming, IPTV, LMS). Published more than 150 papers, 5 books (author, co-author.); Chair of many International Symposiums and conferences, editor conference Proceedings. Since 1999 involved into Cisco Networking academy program in Slovakia as an instructor (CCAI certification) and since 2001 in position of coordinator of the Program in Slovakia (network of 65 universities and secondary schools), 2008-2014 Regional Lead for Russia, Ukraine and CIS. He is a head of the Application Section of the Communication Technology Forum Association in Slovakia, head of Committee on business - academic cooperation, American Chambers of Comers in Slovakia and general manager of University Centre for Innovation, Technology Transfer and Intelectual Property Protection at Technical university of Kosice. He was awarded as a "IT person of the year 2006" in Slovakia 